\title{
ANÁLISIS DE LA PRESENCIA NATURAL DE MICORRIZAS EN CULTIVOS DE ALGODÓN (Gossypium barbadense L.) INOCULADOS CON Bacillus megaterium Y/O Bradyrhizobium yuanmingense
}

\section{ANALYSIS OF NATURAL MYCORRHIZATION IN COTTON CROPS (Gossypium barbadense L.) INOCULATED WITH Bacillus megaterium AND/OR Bradyrhizobium yuanmingense}

\author{
Claudia Valencia ${ }^{1}$ y Doris Zúñiga ${ }^{1}$
}

\begin{abstract}
Resumen
Para el proceso de optimización de protocolos se probaron diferentes modificaciones del proceso de tinción propuesto por Phillips \& Hayman para permitir la identificación de estructuras intraradicales derivadas de la interacción planta-hongo; para ello, se extrajeron las raíces de cultivos de algodón (Gossypium barbadense L.) inoculados con Bacillus megaterium (B), Bradyrhizobium yuanmingense $(\mathrm{Br})$ y la interacción de ambos (I), además los controles positivo, con nitrato de potasio, $(\mathrm{N}+)$ y negativo, sin inoculación, $(\mathrm{N}-)$. Una vez optimizado el protocolo se realizó un conteo de segmentos para calcular el porcentaje de infección natural por Micorrizas Arbusculares (MA) en relación a los tratamientos de fertilización usados. Se encontró que seis de las nueve modificaciones realizadas permiten identificar la presencia de micorrizas en las raíces de algodón, pero la variación D logra una mejor visualización de las características morfológicas de las MA. Se observó también que el \%LRC es bajo (28.77\%) en $\mathrm{N}+$, mientras que la colonización micorrícica se promueve cuando las plantas son inoculadas con B, alcanzando en promedio un 70.98\%. La optimización de los protocolos permite aminorar los costos en la investigación y la contaminación por el uso de reactivos cancerígenos; del mismo modo, encontrar una relación entre las rizobacterias promotoras del crecimiento de las plantas (PGPR) y las MA nos ayuda a identificar nuevas formas de inoculación, lo que permite aumentar la producción.

Palabras clave: B. megaterium, B. yuanmingense, PGPR, MA, G. barbadense $L$.
\end{abstract}

\begin{abstract}
For the process of protocol optimization, different staining process modifications proposed by Phillips \& Hayman, which allow identification of intra-radicals structures derived from the plantfungus interactions, were tested. For the said purpose, the roots of cotton plants (Gossypium barbadense L.) were extracted. These plants were previously inoculated with Bacillus megaterium (B), Bradyrhizobium yuanmingense (Br) separately and as well as in combination (I). In addition to this, a positive control with potassium nitrate $\left(\mathrm{N}^{+}\right)$and without it $(\mathrm{N}-)$ were also included. After the optimization of the staining procedure a count of the segments procedure was carried out to estimate the prevalence of infection by Arbuscular mycorrhizal (AM) in relation to the kind of fertilizers utilized. We found that six of the nine staining modifications allow the identification of the presence of mycorrhiza in roots of cotton plants, however, the variation $\mathrm{D}$ provides a better visualization of the morphological characteristics of AM. It was also noticed that the \% LRC was low i.e. $28.77 \%$ in $\mathrm{N}+$, while the mycorrhizal colonization was most favored when plants were inoculated with $\mathrm{B}$, reaching an average of 70.98\% LRC. Optimizations of standard protocols help reduce the cost and the exposure by the carcinogenic reagents. In conclusion, finding a relationship between AM and plant growth promoting rhizobacteria (PGPR) will help us to identify new ways of inoculation to increase production.
\end{abstract}

Key words: B. megaterium, B. yuanmingense, PGPR, AM, G. barbadense L.

\section{Introducción.}

Los hongos formadores de micorrizas arbusculares (HMA) constituyen el tipo de simbiontes más extendidos en la naturaleza siendo capaces de establecer asociaciones con el 80 a $90 \%$ de plantas vasculares, han beneficiado muchas especies importantes en la agricultura al incrementar su adaptación a diferentes ambientes y con efectos positivos sobre la productividad del sistema (Cuenca et al., 2007; Castillo, 2009). El estudio de la simbiosis micorriza-planta adquiere particular importancia en el trópico debido a que la mayoría de suelos de esta zona 
presentan bajos niveles de fosforo. Como alternativa de solución se ha demostrado que la selección de cepas de HMA eficientes y su producción masiva a escala industrial pueden contribuir en el éxito de la repoblación forestal ya que mejoran el crecimiento de diferentes especies forestales así como el balance ecológico de un ecosistema (Cuervo \& Rivas, 2007). Esta simbiosis es benéfica ya que el hongo coloniza la corteza de la raíz para obtener carbono a partir de la planta hospedera, mientras le ayuda a la planta a tomar fósforo y otros nutrientes minerales del suelo (Serralde \& Ramírez, 2004). Por este motivo se han realizado, en los últimos años, investigaciones para determinar el efecto de aislamientos de HMA sobre sistemas de producción agrícola, para así lograr sistemas de producción sostenibles y competitivos (Martinez \& Pugnaire, 2009). Sin embargo, el conocimiento acerca de la ecología de poblaciones nativas y el papel de las condiciones edáficas y climáticas en el establecimiento y efectividad de esta asociación es limitado. Por ello, es necesario realizar el análisis de poblaciones nativas en relación con los ambientes edáficos en los que se desarrollan. La información obtenida de este tipo de evaluaciones puede conducir a un uso adecuado de estos microorganismos como biofertilizantes, optimizando los resultados en cuanto a producción en sistemas agrícolas y recuperación de ambientes degradados (Velandia, 2006).

Para efectuar un estudio adecuado de las asociaciones micorrizicas es necesario usar un método de tinción de raíces que nos permita observar adecuadamente esta simbiosis; actualmente el método más usado es el propuesto por Phillips \& Hayman (1970) del cual se han derivado la mayoría de métodos de tinción convencionales. Estos procedimientos incluyen varias etapas las cuales varían dependiendo del tipo de raíz que presenta la especie. Debido a que no se encontró publicaciones sobre el estudio sobre la detección de HMA en algodón (Gossypium barbadense var. Tangüis) fue necesario realizar la optimización de un protocolo que permita una mejor visualización de la asociación simbiótica en este cultivo. Una vez optimizado el protocolo se realizó un conteo de segmentos para calcular el porcentaje de infección natural por MA en relación a los diferentes tratamientos utilizados.

\section{Materiales y métodos.}

Toma de muestra.
Las muestras de algodón fueron tomadas a los 180 días, de un ensayo realizado en plantas de algodón a nivel de invernadero en el Laboratorio de Fertilidad de la Facultad de Agronomía - Universidad Nacional Agraria La Molina; como sustrato para las plantas se utilizó una mezcla de suelo:arena (2:1), resultando de ello un suelo franco arenoso ligeramente salino con bajo porcentaje de materia orgánica y alto contenido de fosforo y potasio (Tabla 1). En dicho ensayo se probaron dos inóculos, uno perteneciente a la cepa Bradyrhizobium yuanmingense LMTR28 (Br) aislada del cultivo de pallar (Ormeño-Orrillo et al. 2006) y la otra cepa Bacillus megaterium LMTB5613 (B) aislada del cultivo de algodón ambas de la zona de Ica (Zúñiga 2010); dichas cepas tenían capacidad PGPR probada en el cultivo de algodón (Zuñiga 2010, 2011). Los tratamientos usados fueron: $\mathrm{B}, \mathrm{Br}$ y la interacción de ambos (I), además los controles positivo, con nitrato de potasio, $(\mathrm{N}+)$ y negativo, sin inoculación, $(\mathrm{N}-)$; en tres diferentes momentos de inoculación: a la siembra (M1), en la aparición del hipocótilo (M2) y en ambos momentos (M12); en cada caso se realizaron 4 repeticiones. La parte aérea de la planta fue usada para realizar los siguientes análisis: evaluación del porcentaje de germinación, porcentaje de emergencia y altura de planta a los 30, 60, 90 y 120 días, la cosecha se realizó a los 180 días midiéndose además de los parámetros ya mencionados el peso fresco, peso seco y análisis de nutrientes (datos no mostrados). (Zúñiga, 2011).

Tinción y Preparación de las micorrizas para el estudio microscópico

Las raíces extraídas fueron lavadas con abundante agua hasta eliminar la tierra adherida a la raíz, se dejaron secar a temperatura de ambiente y se cortaron en segmentos de aproximadamente $2 \mathrm{~cm}$., para la tinción se usaron los protocolos descritos por Phillips \& Hayman (1970), Kormanik \& McGraw (1982) y Koske \& Gemma (1989) y las modificaciones propuestas por Pitet et al. (2009) y Chávez et al. (2013). En síntesis, los métodos implementan el uso de hidróxido de potasio $(\mathrm{KOH})$ para el proceso de clarificación de las raíces, seguido de una acidificación con ácido clorhídrico ( $\mathrm{HCl})$ o ácido acético $\left(\mathrm{CH}_{3} \mathrm{COOH}\right)$ y el uso de azul de tripán como colorante. Para determinar el protocolo de tinción para la observación óptima de estructuras micorrícicas en raíces de algodón, se realizaron nueve tratamientos (Tabla 2) en los que se modificaron las concentraciones

Tabla 1. Caracterización del Suelo de la molina

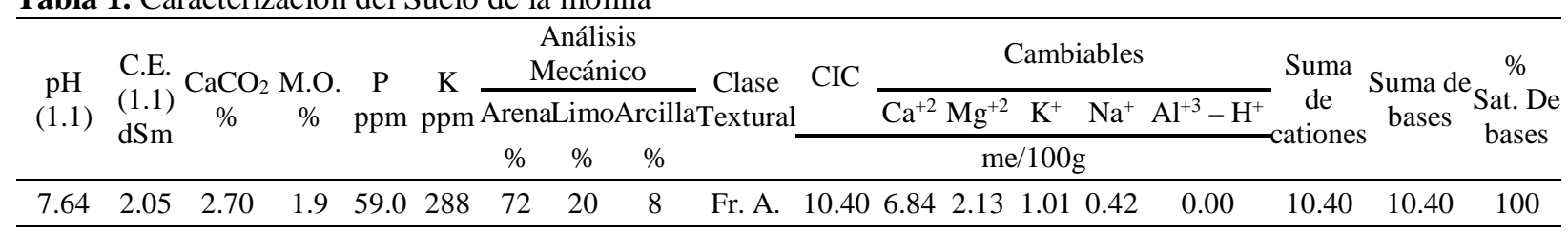

Fr. A.: franco arenoso 
Tabla 2. Diseño experimental para la optimización del proceso de tinción de las raíces de algodón colonizadas con Micorrizas Arbusculares.

\begin{tabular}{|c|c|c|c|c|}
\hline Clarificación & Pre tinción & Tinción & Decoloración & Imagen \\
\hline $\mathrm{KOH} 10 \% 30 \min 65^{\circ}$ & $\mathrm{HCl} 10 \% 20 \mathrm{~min}$ & $15 \min 65^{\circ}$ & Lacto glicerol & $\mathrm{A}$ \\
\hline $\mathrm{KOH} 10 \% 60 \min 65^{\circ}$ & $\mathrm{HCl} 10 \% 20 \mathrm{~min}$ & $15 \min 65^{\circ}$ & Lacto glicerol & B \\
\hline $\mathrm{KOH} 10 \% 90 \min 65^{\circ}$ & $\mathrm{HCl} 10 \% 20 \mathrm{~min}$ & $15 \min 65^{\circ}$ & Lacto glicerol & $\mathrm{C}$ \\
\hline $\mathrm{KOH} 10 \% 120 \min 65^{\circ}$ & $\mathrm{HCl} 10 \% 20 \mathrm{~min}$ & $15 \min 65^{\circ}$ & Lacto glicerol & $\mathrm{D}$ \\
\hline $\mathrm{KOH} 10 \% 15 \min 90^{\circ}$ & $\mathrm{HCl} 1 \% 10 \mathrm{~min}$ & Durante la noche $\mathrm{T}^{\circ}$ Ambiente & Lacto glicerol & $\mathrm{E}$ \\
\hline $\mathrm{KOH} 1 \%$ durante la noche & $\mathrm{HCl} 1 \% 10 \mathrm{~min}$ & Durante la noche $\mathrm{T}^{\circ}$ Ambiente & Lacto glicerol & $\mathrm{F}$ \\
\hline $\mathrm{KOH} 1 \%$ durante la noche & - & Durante la noche $\mathrm{T}^{\circ}$ Ambiente & Lacto glicerol & G \\
\hline $\mathrm{KOH} 1 \%$ durante la noche & Ácido acético $1 \% 5 \mathrm{~min}$ & Durante la noche $\mathrm{T}^{\circ}$ Ambiente & Lacto glicerol & $\mathrm{H}$ \\
\hline $\mathrm{KOH} 1 \%$ durante la noche & Ácido acético 1\% 10 min & Durante la noche $\mathrm{T}^{\circ}$ Ambiente & Lacto glicerol & I \\
\hline
\end{tabular}

de los reactivos utilizados, la temperatura y el tiempo de exposición de las raíces a estos.

\section{Clarificación}

Se pesó 1 gramo de muestra y se adicionó $\mathrm{KOH}$ en diferentes concentraciones (1\% y 10\%) hasta que todas las raíces quedaron cubiertas. Se probaron 3 temperaturas: $25^{\circ} \mathrm{C}$ (temperatura ambiente), $65^{\circ} \mathrm{C}$ y $90^{\circ} \mathrm{C}$ a diferentes tiempos (Tabla 2). Luego se decantó la muestra y se procedió a lavar con abundante agua destilada.

\section{Pre-tinción}

Se adicionó $\mathrm{HCl}$ al $1 \%$ por $10 \mathrm{~min} ., \mathrm{HCl}$ al $10 \%$ por 20 min. y $\mathrm{CH}_{3} \mathrm{COOH}$ al $1 \%$ por 5 y $10 \mathrm{~min}$. tal como se describe en la Tabla 2, pasado el periodo de neutralización se procedió a decantar.

Tinción y decoloración

Se agregó azul de tripán en lactofenol hasta cubrir las raíces; para los tratamientos con $\mathrm{HCl}$ al 10\% se dejó en baño María a $65^{\circ} \mathrm{C}$ por 15 minutos y para los tratamientos con $\mathrm{HCl}$ al $1 \%$ y $\mathrm{CH}_{3} \mathrm{COOH}$ se dejaron durante la noche a temperatura ambiente y se decantó, luego se lavó con lactoglicerol, luego se dejó reposar por 8 días.

\section{Observación microscópica}

Se montaron las raíces en placas de vidrio con unas gotas de lactoglicerol, este último es utilizado para la decoloración y conservación de las raíces; observándose, luego al microscopio óptico algunas de las principales estructuras de la micorrizas, tales como vesículas, arbúsculos, hifas a un lente objetivo de $10 \mathrm{X}$ (algunos casos 40X para mejor observación de las estructuras) LEICA DM750.

Cálculo del porcentaje de colonización

Se tomó una lámina porta objetos y se trazaron cuatro líneas paralelas de forma longitudinal, luego el material coloreado fue cortado en trozos de 1 hasta 2 cm y fue colocado paralelamente sobre el portaobjetos. Las observaciones se realizaron con un lente objetivo de $4 \mathrm{x}$ y se contaron los campos colonizados (Sieverding, 1983). Se calculó el porcentaje de Longitud de Raíz Colonizada (\%LRC) usando la siguiente fórmula:

$$
\% L R C=\frac{\text { Numero de segmentos colonizados }}{\text { Numero total de segmentos contados }}
$$

Análisis estadístico del porcentaje del porcentaje de Longitud de Raíz Colonizada

Los datos del \%LRC fueron analizados con el software estadístico STATGRAPHICS centurión XV.I, para ello se agruparon en cinco tratamientos tomando en cuenta el tipo de inóculo usado y los tres momentos de aplicación dados en la investigación. Estos datos fueron sometidos a un análisis de varianza de clasificación simple y múltiple (ANOVA), siendo los tratamientos con diferencias significativas comparadas según la prueba de rangos múltiples LSD con un nivel de significación del 95\%.

\section{Resultados y discusión.}

Se encontró que seis (D, E, F, G, H, I) de las nueve modificaciones realizadas para el proceso de tinción (Tabla 2) permiten identificar la presencia de micorrizas en las raíces de algodón (Figura 1) habiéndose observado que el tratamiento $\mathrm{D}$ permite una mejor visualización de las características morfológicas de las MA (vesículas y arbúsculos) a pesar de que la temperatura utilizada fue menor $\left(65^{\circ} \mathrm{C}\right)$ que el protocolo original descrito por Phillips \& Hayman (1970). En esta variación de la técnica se empleó un mayor tiempo de exposición a los reactivos y una mayor concentración de $\mathrm{KOH} \mathrm{(10 \% ),} \mathrm{este}$ resultado guarda relación con lo publicado por Pitet et al. (2009) quienes indican que la calidad de la tinción de las MA está vinculada a una buena digestión de las paredes celulares, lo que se logra aumentando la concentración de $\mathrm{KOH}$.

En la etapa de neutralización se observó que tanto las muestras tratadas con $\mathrm{HCl}, \mathrm{CH}_{3} \mathrm{COOH}$ y sin ácido presentaron coloración. Sin embargo en las modificaciones $\mathrm{F}$ e I se pueden diferenciar claramente las raíces de las micorrizas en comparación del resto de tratamientos. Este resultado demuestra la importancia de la etapa de neutralización en el proceso de tinción, ya que permite la penetración del colorante; esto indica que tanto el ácido clorhídrico como el ácido acético pueden ser utilizados sin distorsionar la calidad de la tinción (Ishii \& Loynachan, 2004).

El método usado para el proceso de tinción determina el tipo de estructuras que pueden ser observadas, es así que la tinción con azul de tripán 


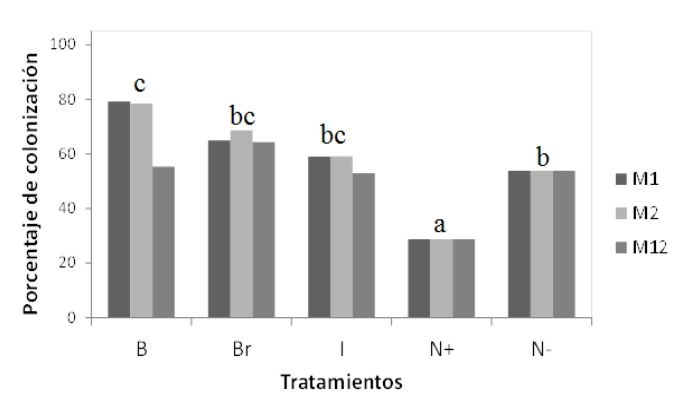

Figura 1. Efecto de los tratamientos (B: $B$. megaterium, Br: B. yuanmingense, I: Interacción B + Br y dos controles N+: Con Nitrógeno y N-: Sin Nitrógeno) en el porcentaje de colonización. M1: inoculación realizada al momento de la siembra, M2: inoculación realizada cuando de observa la presencia del hipocótilo y M12: inoculación realizada al momento de la siembra y cuando de observa la presencia del hipocótilo.

(Phillips \& Hayman, 1970) permite determinar el porcentaje de infección y la densidad visual de MA.

En cuanto al porcentaje de colonización; el análisis de varianza multifactorial (ANOVA) mostró que el factor "tipo de inóculo" tuvo un efecto altamente significativo sobre el \%LRC ( $p=0.0001)$, mas no el factor "momento de inoculación”. Se puedo observar que el porcentaje de infección natural por hongos micorrícicos (N-) fue de 53.81\%, el cual es un indicador de que el algodón es sensible a la infección por micorrizas $y$ se vio afectado negativa $y$ significativamente (28.77\%) por la adición de nitrato de potasio (tratamiento $\mathrm{N}+$ ) (Figura 2); en contraste Cornejo et al. (2008) encontraron que la adición de $\mathrm{NO}^{-}$ produce la alcalinización del suelo lo que conlleva a un aumento en el porcentaje de colonización, pudiendo sin embargo ser alterado por otros factores como el $\mathrm{pH}$ inicial del suelo o el contenido de fosforo (PeñaVenegas et al., 2007). Se observó también que las raíces inoculadas con $B$. megaterium incrementaron significativamente el \%LRC alcanzando un 70.98\%, según lo indicado por Jaizme et al. (2006) las interacciones PGPRs y las MA son muy específicas y pueden, bajo ciertas condiciones, ser capaces de promover la germinación de las esporas y la elongación del tubo germinativo e incluso mejorar la densidad de hifas.

\section{Conclusiones.}

El protocolo que permite una mejor visualización de estructuras micorrícicas en raíces de algodón es el protocolo D. El uso de nitrato de potasio como fertilizante en el cultivo de algodón disminuye el \%LRC (28.77\%) mientras que las raíces inoculadas con B. megaterium promueven la colonización micorrícica, incrementando este valor 70.98 en comparación al control sin inocular; así mismo, el momento de

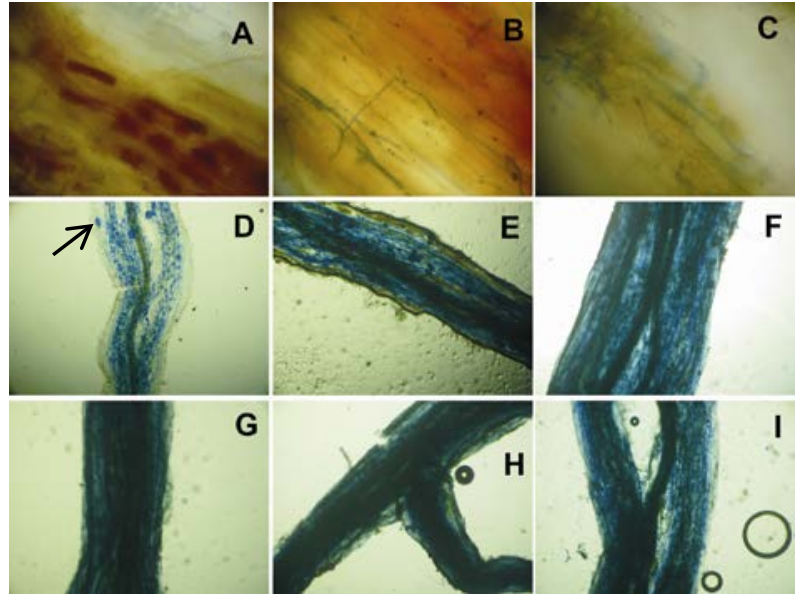

Figura 2. Raíces de algodón teñidas siguiendo el protocolo detallado por Phillips \& Hayman (1970) tomando en cuenta las modificaciones usadas en la Tabla 2. La flecha indica la presencia de vesículas.

aplicación del inoculo no tiene una influencia sobre el \%LRC.

\section{Agradecimientos.}

Proyecto PROTEC 278-2009-CONCYTEC-OAJ.

\section{Literatura citada.}

Castillo M. L. 2009. Caracterización morfológica de micorrizas arbusculares asociadas en raíces de tomate de árbol silvestre (Solanum cajanumensis) y cultivado (Solanum betacea) en dos sectores de la provincia de Loja. Loja, Ecuador: Universidad Técnica Particular de Loja.

Chávez A.T., Lua J., Salmerón I.A., García P.A., Bárcenas A.E. \& Olalde V. 2013. A modified staining technique for the anatomical observation of mycorrhizal roots of woody trees. African Journal of Microbiology Research. 7(28): 3589-3596.

Cornejo P., Rubio R. \& Borie F. 2008. Effect of nitrogen source on some rhizospheric properties and persistence of mycorrhizal fungal propagules in an andisol. Chilean Journal of Agricultural Research. 68 (2): 119-127.

Cuenca G., Cáceres A., Oirdobro G., Hasmy Z. \& Urdaneta C. 2007. Las micorrizas arbusculares como alternativa para una agricultura sustentable en áreas tropicales. Interciencia. 32 (1): 23-29.

Cuervo J. \& Rivas G. 2007. Cuantificación de hongos micorrícicos en muestras de suelo en plantaciones de Tabebuia rosea y Cordia alliodora. NOVA- Publicación científica. 5 (7): 28-41.

Ishii S. \& Loynachan T. 2004. Rapid and reliable DNA extraction techniques from trypan-blue-stained mycorrhizal roots: comparison of two methods. Mycorrhiza. 14 (4):271-275.

Jaizme M., Rodríguez A. \& Barroso L. 2006. Effect of the combined inoculation of arbuscular mycorrhizal fungi and plant- growth promoting rhizobacteria on papaya (Carica papaya L.) infected with the root-knot nematode Meloidogyne incognita. Fruits. 61 (3):1-7.

Kormanik P. \& McGraw A. 1987. Quantification of vesicular-arbuscular mycorrhizae in plant roots. In: 
Schenck NC (ed) Methods and principles of mycorrhizal research. American Phytopathological Society. 37-45.

Koske R. E. \& Gemma J. N. 1989. A modified procedure for staining roots to detect VA mycorrhizas. Mycological Research. 92: 486-489.

Martinez L. B. \& Pugnaire F. I. 2009. Interacciones entre las comunidades de hongos formadores de micorrizas arbusculares y de plantas. Algunos ejemplos en los ecosistemas semiáridos. Ecosistemas. 18 (2): 44-54.

Ormeño-Orrillo E., Vinuesa P., Zúñiga-Dávila D. \& Martínez-Romero E. 2006. Molecular diversity of native bradyrhizobia isolated from Lima bean (Phaseolus lunatus L.) in Peru. Systematic and Applied Microbiology 29: 253-262.

Peña-Venegas C., Cardona G. Arguelles J. \& Arcos A. 2007. Micorrizas arbusculares del sur de la amazonia colombiana y su relación con algunos factores fisicoquímicos y biológicos del suelo. Acta Amazónica. 37(3): 327 - 336

Pitet M., Camprubí A., Calvet C. \& Estaún V. 2009. A modified staining technique for arbuscular mycorrhiza compatible with molecular probes. Mycorrhiza. 19(2): 125-131.

Phillips J. \& Hayman D. 1970. Improved procedures for clearing roots and vesicular-arbuscular fungi for rapid assessment of the infection. Transactions of the British Mycological Society. 55: 158-161.

Serralde A. M. \& Ramírez M. M. 2004. Análisis de poblaciones de micorrizas en maíz (Zea mays) cultivado en suelos ácidos bajo diferentes tratamientos agronómicos. Revista Corpoica. 5 (1):31-40.

Sieverding E. 1983. Manual de métodos para la investigación de la micorriza vesículo arbuscular en el laboratorio. Centro Internacional de Agricultura Tropical. Cali, 123.

Velandia D. L. 2006. Evaluación y Caracterización de Micorrizas Arbusclares Asociadas a Yuca (Manihot esculenta sp.) en dos Regiones de la Amazonía Colombiana. Microbiólogo Agricultura y Veterinaria Pontificia Universidad Javeriana. 24-25.

Zuñiga D. E. 2010. Uso de bacterias promotoras de Crecimiento vegetal para la producción orgánica de cultivos nativos de algodón y pallar en el Valle de Ica. Informe final Proyecto PROTEC 249-2008CONCYTEC-OAJ

Zuñiga D. E. 2011. Aplicación de biofertilizantes para la producción orgánica de cultivos nativos de algodón y pallar en el Valle de Ica. Informe final Proyecto PROTEC 278-2009-CONCYTEC-OAJ. ${ }^{1}$ Laboratorio de Ecología Microbiana y Biotecnología, Departamento de Biología, Facultad de Ciencias,
Universidad Nacional Agraria La Molina, La Molina, Lima, Perú. Correo electrónico dzuniga@lamolina.edu.pe 